\title{
Fabrication of side pump combiners when pumping with a laser diode and a fiber laser
}

\author{
Zhixian Li (李智贤) ${ }^{1}$, Min Fu (付 敏) ${ }^{1}$, Xiaofan Zhao (赵晓帆) ${ }^{1}$, Hongye Li (李宏业 $)^{1}$, Zilun Chen (陈子伦) ${ }^{1,2,3^{*}}$ ， \\ Zefeng Wang (王泽锋 $)^{1,2,3^{* *}}$, and Jinbao Chen (陈金宝) $)^{1,2,3}$ \\ ${ }^{1}$ College of Advanced Interdisciplinary Studies, National University of Defense Technology, Changsha 410073, China \\ ${ }^{2}$ State Key Laboratory of Pulsed Power Laser Technology, Changsha 410073, China \\ 3 Hunan Provincial Key Laboratory of High Energy Laser Technology, Changsha 410073, China \\ *Corresponding author: zilun2003@163.com \\ **Corresponding author: zefengwang_nudt@163.com \\ Received July 2, 2021 | Accepted August 17, 2021 | Posted Online October 8, 2021
}

\begin{abstract}
Side pumping combiners are widely used in fiber laser schemes for their high coupling efficiency, low insertion loss, and multi-point pumping capability. However, side pumping combiners perform differently in coupling efficiency when pumping with a laser diode (LD) and a high-brightness $1018 \mathrm{~nm}$ Yb-doped fiber laser (YDFL). In this paper, for the first time, to the best of our knowledge, we investigated the different parameters to fabricate the $(2+1) \times 1$ combiner with high coupling efficiency when pumping with an LD and a YDFL, respectively. After optimization, the maximum coupled pump power from one single-pump port of the combiner was $1200 \mathrm{~W}$ and $2730 \mathrm{~W}$ when pumping with a LD and a YDFL, respectively.
\end{abstract}

Keywords: side pumping; fiber laser; fiber combiner.

DOI: 10.3788/COL202220.021401

\section{Introduction}

Fiber laser sources have attracted more and more attention due to their high conversion efficiency, good beam quality, compact structure, and convenient thermal management ${ }^{[1-3]}$. As the crucial component of fiber lasers, a pump source can be mainly divided into laser diode (LD) pumping and $\mathrm{Yb}$-doped fiber laser (YDFL) pumping, wherein the YDFL pumping has the advantages of low quantum deficit and high optical efficiency ${ }^{[4,5]}$. Nowadays, the power scaling of high-power fiber laser systems is mainly limited by stimulated Raman scattering (SRS), stimulated Brillouin scattering (SBS), and transverse mode instability (TMI). Facts have proved that counter-pumping or bidirectional pumping schemes can help increase the threshold of SRS and TMI in fiber laser systems, thereby increasing the output power ${ }^{[6,7]}$. Side pumping combiners have many advantages when being used in the counter-pump scheme. First, the pump power is coupled through the side of the signal fiber, which can help to realize a multi-point cascade pump structure and improve the pumping capability of the laser system. Further, the signal fiber is uninterrupted throughout the manufacturing process, which is helpful to reduce the insertion loss and keep good beam quality. In addition, the high reverse pump isolation value of the side pump combiner can protect the pump source from power back propagation, making it more suitable for the reverse pump or bidirectional pump scheme ${ }^{[8]}$.
Side pumping technology can be divided into non-all-fiber pumping technology and all-fiber pumping technology. Nonfull fiber side pumping technology is usually achieved with the help of complex optical devices such as adhesive microprisms $^{[9]}, \mathrm{V}$-grooves ${ }^{[10]}$, embedded mirrors ${ }^{[11]}$, and diffraction gratings ${ }^{[12]}$. However, due to the low damage threshold and complex spatial structure of these optical facilities, the stability, coupling efficiency, and power handling capabilities of this pumping technology are relatively low.

Among the all-fiber structure of the side pump technology, the combiner fabricated with taper-fused method is considered to be the key element to realize the high-power fiber laser due to the high efficiency, high-power processing capability, and low signal loss. The side pumping combiner based on the taper-fused method was reported in previous research for both LD pumping and YDFL tandem pumping structures. For the LD pumping scheme, in 2012, Theeg et al. reported a $(4+1) \times 1$ combiner with the maximum handling power of about $110 \mathrm{~W}$ for each pump port ${ }^{[13]}$. Later in 2013, Tsinghua University reported a $(2+1) \times 1$ refractive valley configuration side pump combiner to protect the whole system from the backward pump light. The maximum coupled pump power from each pump port was about $200 \mathrm{~W}$ with the efficiency of $96 \%{ }^{[14]}$. In 2015 , Chen et al. studied the relationship between coupling efficiency and fabrication parameters such as taper length of pump fiber for 
a $(2+1) \times 1$ side pumping combiner. After optimization, the maximum coupled power from one pump port was about $600 \mathrm{~W}$ with the coupling efficiency of about $98.6 \%$. Further, In 2016, Tan et al. reported a multi-point side pump structure using five $(1+1) \times 1$ side pumping combiners to construct a bidirectionally pumping fiber laser oscillator, each of which is coupled with $150 \mathrm{~W}$ pump power ${ }^{[15]}$. Later, Dong et al. applied this multi-point pumped structure directly in the $\mathrm{Yb}$-doped gain fiber. This unique pumping scheme helps to reduce the temperature of the gain fiber by $11^{\circ} \mathrm{C}$ with $110 \mathrm{~W}$ pump power injected from each pump port when compared with using the end pumping combiner under the same power level ${ }^{[16]}$. In 2018, Xiao et al. used two $(6+1) \times 1$ combiners to build the bidirectional LD pumping configuration, each of which achieved $3.6 \mathrm{~kW}$ coupled pumping power ${ }^{[17]}$. For YDFL tandem pumping, our group reported a co-directional amplifier based on home-made side pumping combiner in 2017, in which the maximum coupled pump power from one pump port was about $1.5 \mathrm{~kW}^{[18]}$. Recently, Wang et al. reported a $5.1 \mathrm{~kW}$ tandem pumped amplifier; in their experimental setup, a $(3+1) \times 1$ combiner was used to couple $5.6 \mathrm{~kW} 1018 \mathrm{~nm}$ YDFL pump power into the system $^{[19]}$.

In this study, for the first time, to the best of our knowledge, we have separately studied the different manufacturing parameters of the side pumped combiner catering for LD pumping and high-brightness YDFL pumping. Theoretically, the geometrical optics theory was used to analyze the propagation path of pump light with different numerical apertures (NA) in the side pump combiner, which provides a useful reference for the manufacturing process. Experimentally, we have studied the pump coupling efficiency and temperature characteristics of the side pump combiner when it was pumped by an LD and a YDFL. By changing the fusion area between the signal fiber and the tapered pump fiber, a side pump combiner with high coupling efficiency can be manufactured in the case of LD pumping and YDFL pumping, respectively. In the LD pumping case, the maximum coupled pump power from one pump port was $1200 \mathrm{~W}$ with the coupling efficiency of $97 \%$, and the highest temperature rise coefficient of the combiner was $12.41^{\circ} \mathrm{C} / \mathrm{kW}$. In the YDFL pumping case, the maximum coupled pump power from one pump port was $2730 \mathrm{~W}$ with the coupling efficiency of $99 \%$, and the highest temperature rise coefficient of the combiner was $6.5^{\circ} \mathrm{C} / \mathrm{kW}$. The high-power withstanding capacity of the side pumping combiner in both the LD pumping and YDFL pumping schemes can provide a potential method in highpower, bi-directional pumping fiber laser applications.

\section{Theoretical Analysis}

As described in our previous work ${ }^{[20]}$, the geometric optics theory is used to analyze the propagation path of pump light with different NA values in the combiner. As evidenced in Fig. 1, the leakage pump light of the combiner is mainly leakage power at the end of pump fiber (LPE) and leakage power into the coating of signal fiber (LPC) ${ }^{[8]}$. The pump light is transmitting

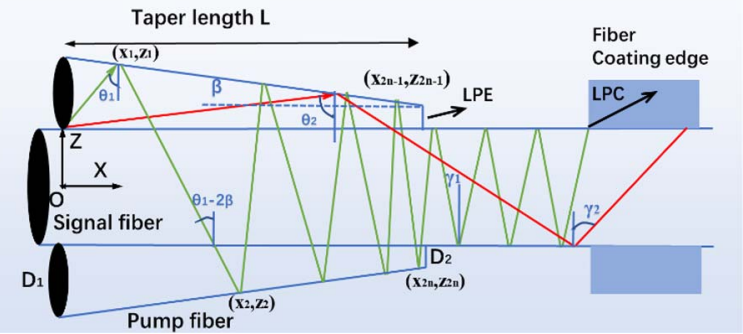

Fig. 1. Pump light with different NAs transmitting in the $(2+1) \times 1$ side pumping combiner.

in the tapered pump fiber with the transmitting angle $\gamma$ reduced by $2 \beta$ after each reflection $\left\{\beta=\arctan \left[\left(D_{1}-D_{2}\right) / L\right]\right.$, where $D_{1}$ is the original pump diameter, and $D_{2}$ is the waist diameter of the tapered pump fiber, while $L$ is the corresponding taper length\}. The value of the final transmitting angle of pump light in the central fiber can be estimated as $\gamma=\theta-2 i \beta$, where $\theta$ is the incident angle, and $i$ is the total reflection number in the tapered area for pump light. For this numerical analysis, the taper length $L$ is set as $2.5 \mathrm{~cm}, D_{1}$ and $D_{2}$ are $220 \mu \mathrm{m}$ and $10 \mu \mathrm{m}$, respectively, and the radius of the signal fiber equals $200 \mu \mathrm{m}$.

When $i=2 n$, with $n$ being the positive integer, the intersection point $\left(x_{i}, z_{i}\right)$ of the pump fiber boundary and the pump light could be calculated through Eqs. (1) and (2), wherein $k_{i}$ is the slope of the incident light transmitting in the combiner:

$$
\begin{gathered}
k_{i}=-\tan [\theta-2(i-1) \beta], \\
\left\{\begin{array}{c}
z_{i}=(\tan \beta) \cdot x_{i}-420 \\
z_{i}-z_{i-1}=k_{i}\left(x_{i}-x_{i-1}\right)
\end{array} .\right.
\end{gathered}
$$

When $i=2 n+1$, the intersection point $\left(x_{i}, z_{i}\right)$ of the pump fiber boundary and the pump light could be calculated through Eqs. (3) and (4):

$$
\begin{gathered}
k_{i}=\tan [\theta-2(i-1) \beta], \\
\left\{\begin{array}{c}
z_{i}=(-\tan \beta) \cdot x_{i}+420 \\
z_{i}-z_{i-1}=k_{i}\left(x_{i}-x_{i-1}\right)
\end{array}\right.
\end{gathered}
$$

Through the iterative method, the propagation paths of the incident light with different NAs could be calculated by using Eqs. (1)-(4). When the value of $x_{i}$ is larger than $L$, the incident pump light is considered to transmit through the coupling area between the pump and central fibers in the combiner. Subsequently, the pump light is transmitting in the central fiber with $\gamma$ remaining unchanged. It is worth noting that those pump rays starting at the junction point of the pump fiber and signal fiber are the latest reflected into the signal fiber. Therefore, to include transmitting rays as many as possible, the approximation $\left(x_{1}, z_{1}\right)=(0,200)$ is used in this analysis.

According to the geometric optical theory, we calculated the transmission trajectory of the incident light with two different NAs in the side pumping combiner, which is shown in Fig. 2. 


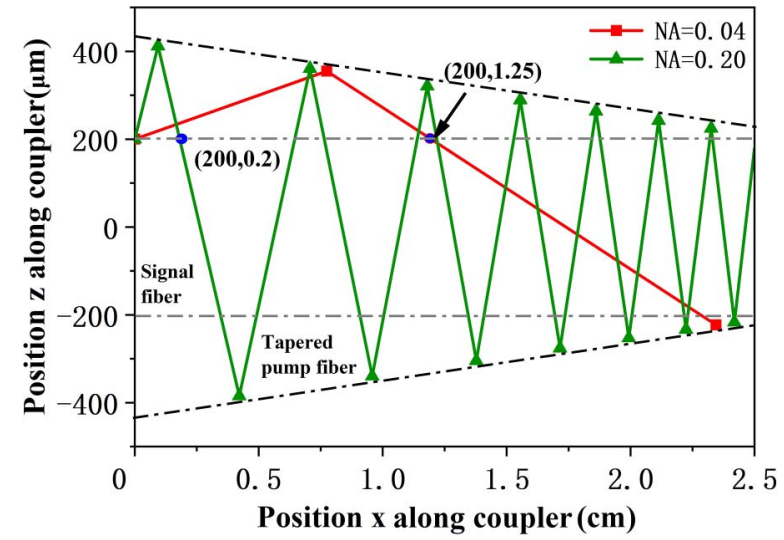

Fig. 2. Transmission paths of pump light with different NAs in the combiner calculated through the geometric method.

As shown in Fig. 2, the green line (pump light with NA =0.2) represents the LD pumping scheme, while the red line (pump light with NA $=0.04$ ) represents the YDFL pumping scheme. As evident in this figure, for pump light with different NAs, the initial coupling position to the signal fiber varies greatly. For example, the initial coupling point of the pump light with $\mathrm{NA}=0.2$ is at $x=0.2 \mathrm{~cm}$, while for the pump light with $\mathrm{NA}=0.04$, this point locates at $x=1.25 \mathrm{~cm}$. Therefore, it is necessary to adjust the fusion area of the combiner to optimize its pump coupling efficiency according to different pumping sources. In addition, the reflection number $i$ at the tapered pump fiber is 14 for the pump light whose NA is 0.2 , while for pump light whose NA equals 0.04 , this number drastically decreases to only two. Therefore, for the side pumping combiner, the use of a high-brightness YDFL pump source can effectively reduce the divergence angle $\gamma(\gamma=\theta-2 i \beta)$ of the pump light coupled into the signal fiber, which is beneficial to improve the pump coupling efficiency and the temperature characteristics under high-power operation.

\section{Experimental Results}

Figure 3 gives the fabrication scheme and end face microscope graph (inserted picture) of a $(2+1) \times 1$ side pump combiner. In this experiment, the pump fiber parameters are $220 / 242 \mu \mathrm{m}$ and $0.22 \mathrm{NA}$, while the parameters of the central fiber are $25 / 400 \mu \mathrm{m}$ and NA $=0.06 / 0.46$, respectively. The detailed introduction of the fabrication process and fabrication system of our homemade combiner was described in our previous work ${ }^{[16]}$. Two pump fibers are tapered and then attached firmly to the surface of the central fiber. The hydrogen-oxygen flame is used to fuse these three fibers together. Here, two different pump sources (YDFL and LD) are spliced with the pump pigtail for online monitoring, respectively. Figure 3(b) illustrates the physical picture of a combiner packaged in a copper fixture: fibers are fixed by ultraviolet adhesive in the groove of the copper fixture for heat dissipation and long-term use.

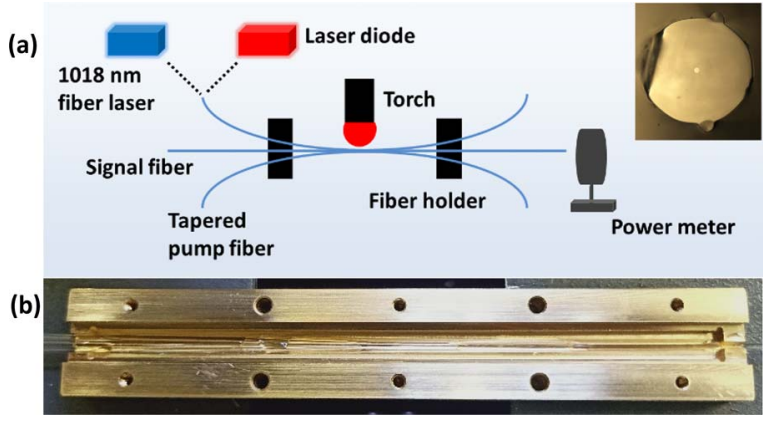

Fig. 3. (a) Schematic diagram of the manufacturing system of the combiner, insert: a side microscope picture of the manufactured side pump combiner; (b) the physical picture of a combiner packaged in copper fixture.

As illustrated in the theoretical analysis, the initial coupling position to the signal fiber for the pump light varies greatly in the LD pumping and YDFL pumping schemes. During the production process, only part of the tapered pump fiber can be spliced to the surface of the central fiber; therefore, the different fusion areas will affect the performance of the combiner under different pumping schemes. During the specific fabrication, the fusion area could be adjusted through changing the attached portion of the tapered pump fibers to the signal fiber and the lateral position of the heating flame. Under the conditions where the fusion time $(60 \mathrm{~s})$, the cone length $(1.8 \mathrm{~cm})$, and the waist diameter $(10 \mu \mathrm{m})$ of the tapered pump fiber remained unchanged, side pump combiners with different fusion areas were prepared. The scanning diagrams of the fusion area of the two side pump combiners are shown in Fig. 4. The black curve is the tapered pump fiber, and the shaded area is the fusion area of the tapered pump fiber and central fiber. Limited by the size of the torch, the length of the fusion zone was kept constant (about $1 \mathrm{~cm}$ ) in the fabrication. In order to verify the correctness of the experiment, two samples were made in each fabrication parameter: the fusion areas of combiner 1 and combiner 2 are illustrated in Fig. 4(a), while the fusion areas of combiner 3 and combiner 4 are illustrated in Fig. 4(b).

The divergence angle of the LD and YDFL pump source used in the test process is measured through a CCD camera (Thorlabs, BC106N-VIS/M), and the corresponding NA is thereafter calculated as 0.17 and 0.05 , respectively. The coupling efficiency and signal insertion loss test results are given in Table 1.
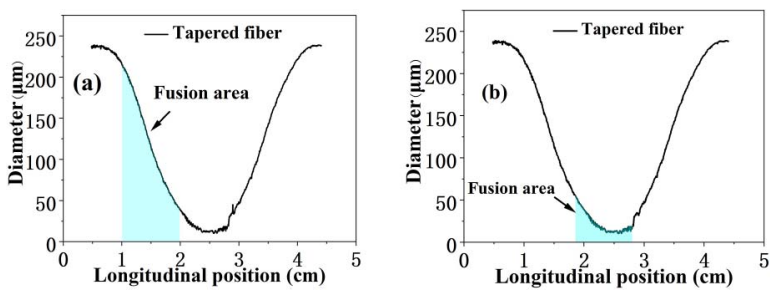

Fig. 4. Scanning diagrams of fusion areas of different side pumping combiner samples; (a), (b) represent the fabrication parameters of combiners designed for LD pumping and YDFL pumping, respectively. 
Due to the relatively low coupling efficiency, the pump power in the test process is lower than $100 \mathrm{~W}$ here for the sake of security, and the high-power testing results will be given in the next section. As evident in Table 1, in terms of pump coupling efficiency, combiners 1 and 2 perform better than combiners 3 and 4 in the case of LD pumping, but in the case of YDFL pumping, the results are just the opposite. The reason why combiners 1 and 2 have a relatively low coupling efficiency in the YDFL pumping scheme is that only the starting end of the tapered pump fiber is spliced into the central fiber. As illustrated in Fig. 2, the high-brightness pump light from the YDFL has not been completely reflected into the signal fiber and will leak as LPE from the pump pigtail, decreasing the coupling efficiency. However, for combiners 3 and 4, the starting end of the tapered pump fiber is partly spliced into the central fiber. Therefore, high NA pump light will be limited in the pump fiber and cannot transmit into the central fiber in this area. Subsequently, within the same longitudinal distance in the combiner, the reflection number for the pump light will increase, so will the divergence angle of the pump light, which will cause more light to leak as LPC and decrease the coupling efficiency. The signal insertion loss of the combiner is measured through a $1064 \mathrm{~nm}$ singlemode fiber laser source. As described in Ref. [21], the insertion loss for the signal fiber of the side pumping combiner is closely related the flame temperature. For combiners 3 and 4 , the waist diameter of the tapered pump fiber in the fusion area is much smaller than that of combiners 1 and 2. In this case, a relatively low flame temperature can fuse the tapered portion into the central fiber and therefore ensure a high coupling efficiency of the combiner when pumping with high-brightness YDFL. Subsequently, smaller impact and insertion loss will be introduced to the signal fiber in the combiner fabricated for YDFL pumping when compared with the combiner fabricated for LD pumping.

As shown in Fig. 5, two LDs (Reci, DAB1200, wavelength 915 and $976 \mathrm{~nm}$ ) were utilized to test the characteristics of combiner 1 in high-power operation. An endcap was spliced with the output signal fiber of combiner 1 in order to decrease the backward light and protect the system. The packaged combiner was placed on a heat sink (keeping temperature of $20^{\circ} \mathrm{C}$ with the assistance of water cooling), and the working temperature of the system was monitored by a thermal imager.

Table 1. Relationship between the Pump Coupling Efficiency and the Signal Insertion Loss of Different Side Pump Combiners with Different Pump Sources.

\begin{tabular}{lccc}
\hline & $\begin{array}{c}\text { LD Pump } \\
\text { Efficiency (\%) }\end{array}$ & $\begin{array}{c}\text { YDFL Pump } \\
\text { Efficiency (\%) }\end{array}$ & $\begin{array}{c}\text { Signal Insertion } \\
\text { Loss (\%) }\end{array}$ \\
\hline Combiner 1 & 97.5 & 84.5 & 3.9 \\
Combiner 2 & 97.0 & 86.3 & 3.4 \\
Combiner 3 & 85.7 & 99.0 & 1.7 \\
Combiner 4 & 84.0 & 98.3 & 1.9 \\
\hline
\end{tabular}

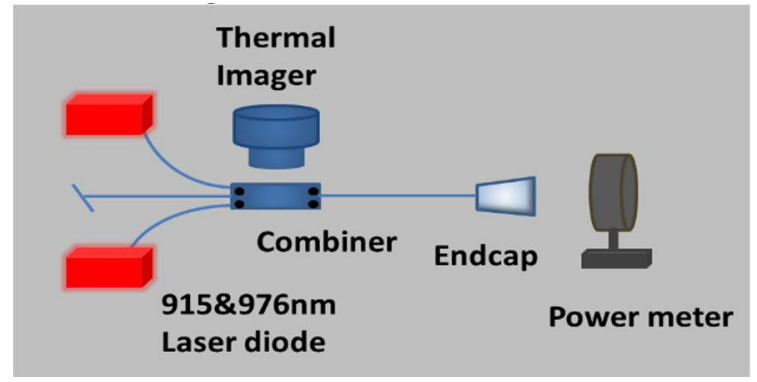

Fig. 5. Experimental setup of the coupling efficiency and temperature characteristic testing system of combiner 1 (with LD pumping).

The testing results of combiner 1 are illustrated in Fig. 6 . As evident in Fig. 6, the output power of the combiner increases linearly with the input pump power, the peak value is $2179 \mathrm{~W}$ when the pump power of $2267 \mathrm{~W}$ is injected, and the corresponding coupling efficiency is about $97 \%$. Moreover, the highest temperature of the side pump combiner also increases as the input pump power increases linearly with a coefficient of $12.41^{\circ} \mathrm{C} / \mathrm{kW}$. The thermal picture inserted in Fig. 6 shows the temperature characteristics of combiner 1 working with 2267 W LD pump power injected. The highest temperature $\left(50^{\circ} \mathrm{C}\right)$ is located at the edge of the coating of the central fiber, which determines the power tolerance of the side pump combiner.

The high-power test scheme of combiner 3 with YDFL pumping is given in Fig. 7, which is similar to Fig. 5, except that the pumping source was replaced by a combined high-brightness $1018 \mathrm{~nm}$ YDFL (each YDFL with a core diameter of $10 \mu \mathrm{m}$ ). The test results are shown in Fig. 8. From this figure, the output power increases linearly with the increase of input power with a coefficient of $99 \%$.

Besides, the highest temperature of the side pumping combiner also increases linearly with the increase of input pump power with a coefficient of $6.5^{\circ} \mathrm{C} / \mathrm{kW}$. It is worth noting that combiner 3 performs better than combiner 1 in terms of pump coupling efficiency and temperature characteristics thanks to the

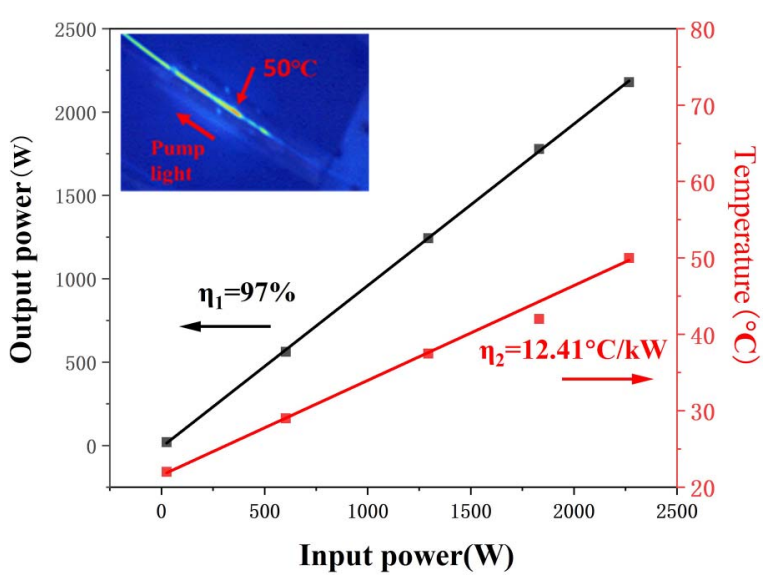

Fig. 6. Results of pump coupling efficiency and temperature characteristics of combiner 1 when pumped with the LD. Insert: the thermal picture of the home-made side pumping combiner when injecting 2267 W LD pump power. 


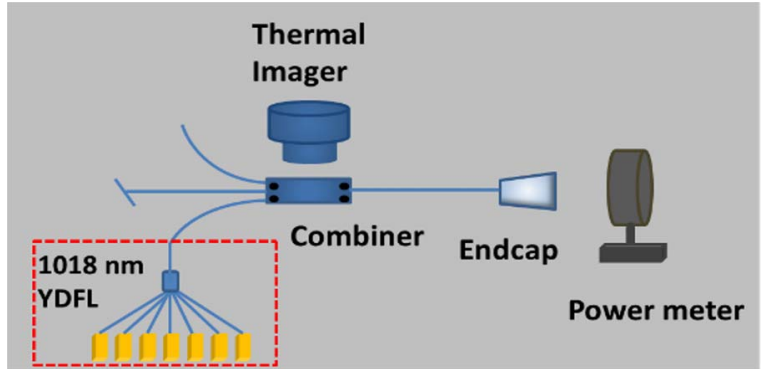

Fig. 7. Experimental setup of the coupling efficiency and temperature characteristic testing system of combiner 3 (with YDFL pumping).

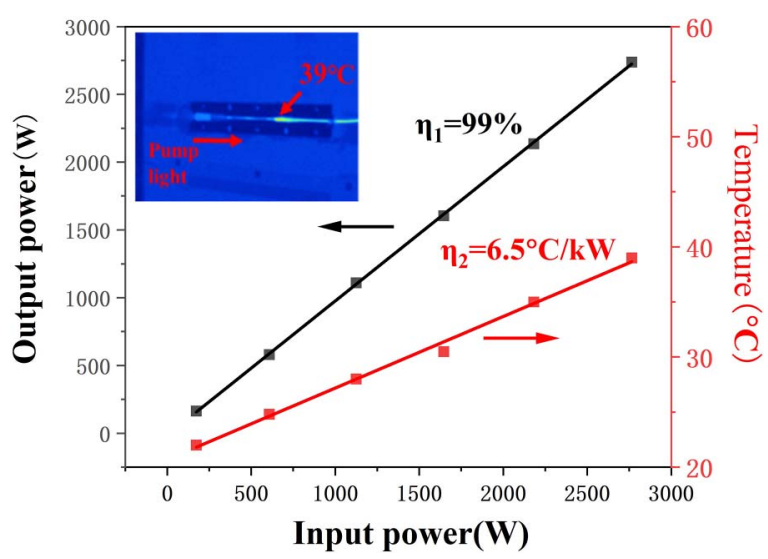

Fig. 8. Results of pump coupling efficiency and temperature characteristics of combiner 3 when pumped with the YDFL. Insert: the thermal picture of the home-made side pumping combiner when injecting 2730 W YDFL pump power.

high-brightness pump source from the YDFL, proving the potential of the pumping capacity improvement through the side pumping combiner and tandem pumping scheme. The picture inserted in Fig. 8 shows the thermal picture of combiner 3 working with $2730 \mathrm{~W}$ YDFL injected from one pump port. Through experimental measurement, the other pump port performs similarly to the previous tested port.

\section{Discussion and Conclusion}

The experiment reveals that first, to improve the coupling efficiency, the fabricated parameters for side pumping combiners designed for LD pumping and YDFL pumping are different: for LD pumping application, the starting end of the tapered pump fiber should be fused into the central fiber, while for YDFL tandem pumping, the waist of the tapered pump fiber should be fused into the central fiber. Second, the pump light from the YDFL has a smaller divergence angle when propagating in the combiner, which will decrease the power because of LPC. Subsequently, the coupling efficiency and temperature characteristics of the side pumping combiner in this case are better than that directly pumped by the LD source. Moreover, for the side pumping combiner designed for YDFL pumping application, the waist size of the tapered pump fiber in the fusion area is much smaller, which means that a low flame temperature during the fusion process is enough to ensure a high coupling efficiency. Thereafter, when compared with the combiner designed for LD pumping, the combiner designed for YDFL pumping performs better in insertion loss and beam quality characteristics of the signal fiber.

In summary, this paper investigated the performance of a $(2+1) \times 1$ side pumping combiner in LD pumping and YDFL pumping, respectively. Through theoretical and experimental analysis, we put up with the optimization method to enhance the coupling efficiency for the side pumping combiner in different pumping schemes. After optimization, the fabricated combiner designed for LD pumping could bear $1200 \mathrm{~W}$ LD pump power from one single port with the coupling efficiency of $97 \%$, and the highest temperature rise coefficient of the combiner was $12.41^{\circ} \mathrm{C} / \mathrm{kW}$. For the combiner designed for the YDFL pumping case, the maximum coupled pump power from one pump port was $2730 \mathrm{~W}$ with the coupling efficiency of $99 \%$, and the highest temperature rise coefficient of the combiner was $6.5^{\circ} \mathrm{C} / \mathrm{kW}$. From our perspectives, those side pump combiners made with optimized parameters show great potential in high-power, bi-directional pumping, and excellent beam quality fiber laser systems.

\section{References}

1. D. J. Richardson, J. Nilsson, and W. A. Clarkson, "High power fiber lasers: current status and future perspectives," J. Opt. Soc. Am. B 27, B63 (2010).

2. S. D. Jackson, "Towards high-power mid-infrared emission from a fiber laser,” Nat. Photon. 6, 423 (2012).

3. H. Chen, X. Jiang, S. Xu, and H. Zhang, "Recent progress in multi-wavelength fiber lasers: principles, status, and challenges," Chin. Opt. Lett. 18, 041405 (2020).

4. H. Xiao, P. Zhou, X. L. Wang, S. F. Guo, and X. J. Xu, "High power $1018 \mathrm{~nm}$ monolithic $\mathrm{Yb}^{3+}$-doped fiber laser and amplifier," Laser Phys. Lett. 9, 748 (2012).

5. Z. Wang, P. Yan, Y. Huang, J. Tian, C. Cai, D. Li, Y. Yi, Q. Xiao, and M. Gong, "An efficient 4-kW level random fiber laser based on a tandem-pumping scheme," IEEE Photon. Technol. Lett. 31, 817 (2019).

6. M. Hu, Z. Quan, J. Wang, K. Liu, X. Chen, C. Zhao, Y. Qi, B. He, and A. Zhou, "Stimulated Brillouin scattering threshold dependent on temporal characteristics in a kilowatt-peak-power, single-frequency nanosecond pulsed fiber amplifier," Chin. Opt. Lett. 14, 031403 (2016).

7. B. Yang, H. Zhang, C. Shi, R. Tao, R. Su, P. Ma, X. Wang, P. Zhou, X. Xu, and Q. Lu, "3.05 kW monolithic fiber laser oscillator with simultaneous optimizations of stimulated Raman scattering and transverse mode instability," J. Opt. 20, 025802 (2018).

8. C. Lei, Z. Chen, J. Leng, Y. Gu, and J. Hou, "The influence of fused depth on the side-pumping combiner for all fiber lasers and amplifiers," J. Lightwave Technol. 35, 1922 (2017).

9. D. Wang, Y. Wang, and S. Liu, "New reflecting side-pumped method of double-clad fiber laser by micro-prism," Acta Opt. Sin. 29, 974 (2009).

10. J. P. Koplow, L. Goldberg, and D. A. V. Kliner, "Compact 1-W Yb-doped double-cladding fiber amplifier using V-groove side-pumping," IEEE Photon. Technol. Lett. 10, 793 (1998).

11. J. P. Koplow, S. W. Moore, and D. A. V. Kliner, "A new method for side pumping of double-clad fiber sources," IEEE J. Quantum Electron. 39, 529 (2003).

12. S. L. Lin, Y. W. Lee, K. Y. Hsu, C. W. Huang, and S. L. Huang, "Design of resonantly side-pumped 1645-nm Er:YAG crystal fiber lasers with grating couplers," in Conference on Lasers and Electro-Optics Pacific Rim (CLEO-PR) (2013), paper TuA1_5. 
13. T. Theeg, H. Sayinc, J. Neumann, L. Overmeyer, and D. Kracht, "Pump and signal combiner for bi-directional pumping of all-fiber lasers and amplifiers," Opt. Express 20, 28125 (2012).

14. Q. Xiao, P. Yan, H. Ren, X. Chen, and M. Gong, "A side-pump coupler with refractive index valley configuration for fiber lasers and amplifiers," J. Lightwave Technol. 31, 2715 (2013).

15. Q. Tan, T. Ge, X. Zhang, and Z. Wang, "Cascaded combiners for a high power CW fiber laser," Laser Phys. 26, 025102 (2016).

16. D. Fanlong, Z. Xinhai, and S. Feng, "Side coupler applied in a multi-pumped Yb-doped triple-clad fiber laser," Laser Phys. 28, 125106 (2018).

17. Q. Xiao, D. Li, Y. Huang, X. Wang, Z. Wang, J. Tian, P. Yan, and M. Gong, "Directly diode and bi-directional pumping $6 \mathrm{~kW}$ continuous-wave all-fibre laser," Laser Phys. 28, 125107 (2018).
18. Y. Gu, C. Lei, J. Liu, R. Li, L. Liu, and H. Xiao, "Side-pumping combiner for high-power fiber laser based on tandem pumping," Opt. Eng. 56, 116109 (2017).

19. Z. Wang, W. Yu, J. Tian, T. Qi, D. Li, Q. Xiao, P. Yan, and M. Gong, "5.1 kW tandem-pumped fiber amplifier seeded by random fiber laser with high suppression of stimulated Raman scattering," IEEE J. Quantum Electron. 57, 6800109 (2020).

20. Z. Li, M. Fu, X. Tian, X. Zhao, H. Li, Z. Chen, Z. Wang, and J. Chen, "Investigation of the pump coupling efficiency of a side-pumping combiner based on tapered-fused method," Opt. Express 29, 17784 (2021).

21. C. Lei, Z. Chen, H. Yang, Y. Gu, and H. Jing, "Beam quality degradation of signal light in a side pumping coupler with a large-mode-area signal fiber," Opt. Express 27, 14041 (2019). 